\title{
Ultrasound-guided transversus abdominis plane injection with computed tomography correlation: a cadaveric study
}

This article was published in the following Dove Press journal: Journal of Pain Research

29 June 2013

Number of times this article has been viewed

\author{
Susan M Moeschler' \\ Naveen S Murthy ${ }^{2}$ \\ Bryan C Hoelzer' \\ Halena M Gazelka' \\ Richard H Rho' \\ Matthew J Pingree ${ }^{1,3}$ \\ 'Department of Anesthesiology, \\ ${ }^{2}$ Department of Radiology, \\ ${ }^{3}$ Department of Physical Medicine \\ and Rehabilitation, Mayo Clinic, \\ Rochester, Minnesota, USA
}

Correspondence: Susan M Moeschler Department of Anesthesiology, Mayo Clinic, 200 First St SW, Rochester, MN 55905, USA

Email moeschler.susan@mayo.edu
Background: Ultrasound-guided transversus abdominis plane (TAP) injections are increasingly being used as an alternative to traditional perioperative analgesia in the abdominal region. With the use of a "blind" TAP block technique, these procedures have had variable success in cadaver and in vivo studies. For more accurate injection with the intended medication, ultrasound guidance allows visualization of the correct layer of the abdominal wall planes in which the thoracolumbar nerves reside.

Objective: To assess the spread of various volumes of contrast placed under live ultrasound guidance into the TAP using computed tomography (CT).

Methods: Four TAP blocks were performed on 2 fresh frozen cadaver torsos with predetermined contrast volumes of $5,10,15$, or $20 \mathrm{~mL}$. A CT scan of the cadaver was then performed and interpreted by a musculoskeletal radiologist. This cadaver study was carried out at a tertiary care academic medical center.

Results: Cranial-caudal spread of injected contrast correlated with increasing injectate volume and was roughly 1 vertebral level (end plate to end plate) for the $5 \mathrm{~mL}$ injection and 2 vertebral levels for the 10, 15, and $20 \mathrm{~mL}$ injections. However, the degree of injectate spread may be different for live patients than for cadavers.

Conclusion: This study helps further the understanding of injectate spread following ultrasoundguided TAP injections. Specifically, it suggests that $15 \mathrm{~mL}$ provides additional cranial-caudal spread and may be an optimal volume of anesthesia.

Keywords: abdominal cadaver study, abdominal computed tomography scan, abdominal wall pain, TAP block, ultrasound-guided injection

\section{Introduction}

Abdominal wall pain is a common complaint, and patients can present with it in the acute perioperative period as well as in a chronic pain state. ${ }^{1}$ Pain-relieving therapies are varied, and range from oral medication to interventional procedures. Recently, the ultrasound-guided transversus abdominis plane (TAP) block has become more widely used as an alternative technique for perioperative analgesia for abdominal procedures. ${ }^{2-4}$

The abdominal wall is innervated by the anterior division of the lower thoracic and upper lumbar nerves. The nerves course within the fascial plane between the internal oblique and transverse abdominis muscle - the TAP. ${ }^{5}$ With this anatomy and its innervation, the TAP block has been shown to provide effective postoperative analgesia, reducing opioid requirements and improving patient satisfaction in the early postoperative period. ${ }^{5}$ 
The TAP block had been used previously with a nonguided or "blind double-pop" technique that relies on tactile sensation to determine the appropriate level of local anesthetic infiltration. ${ }^{6}$ Cadaver studies have established the Petit triangle, defined medially by the latissimus dorsi muscle, laterally by the external oblique muscle, and inferiorly by the iliac crest. These superficial landmarks, when combined with knowledge of the path of the thoracolumbar nerves, have allowed clinicians to perform blind TAP blocks with varying degrees of success. ${ }^{7,8}$ There are 2 potential pitfalls with a blind TAP block. First, the final needle tip position may be superficial to the transversus abdominis fascial plane, resulting in intramuscular or subcutaneous deposition of the injectate. Second, the final needle tip position may be deep to the intended target, resulting in an intraperitoneal injection. Both of these aberrant injections would produce a failed block that results in pain, requiring rescue pain management because of liver laceration or bowel injury, to name just a few complications. ${ }^{9}$ With the advent of ultrasound guidance, the transversus abdominis fascial plane in which the thoracolumbar nerves reside can be identified and accurately injected with the intended medication. ${ }^{10,11}$

The ideal volume for a TAP block is unknown. Different techniques (ie, subcostal versus midaxillary) have been described, and one study suggests that the more subcostal TAP compartment does not communicate with the more lateral triangle of Petit. ${ }^{12}$ Volumes of injectate have varied among studies, with some using weight-based volumes versus standard volumes, without any conclusions regarding optimal volume. A recent retrospective review by Abdallah et $\mathrm{al}^{13}$ suggested that $15 \mathrm{~mL}$ may be an optimal volume, although findings were inconclusive. We therefore sought to further investigate the spread of various volumes of contrast injected under ultrasound guidance into the TAP layer as identified by computed tomography (CT) imaging.

\section{Methods}

After receiving approval from the Mayo Clinic Institutional Review Board, we obtained two unembalmed adult cadaveric torso specimens. The anatomical specimens were fully thawed at room temperature immediately before the study. No specimens exhibited signs of prior surgery, trauma, or major deformity around the abdomen, specifically at the injection sites. Each cadaver was imaged with a 64-slice $(2 \times 32$ detectors) Siemens SOMATOM Definition CT scanner (Siemens Healthcare, Erlangen, Germany) using a scan protocol consisting of 2-mm axial sections obtained in a softtissue kernel. All images were interpreted by an experienced musculoskeletal radiologist using a GE Advantage Workstation (GE Healthcare Institute, Waukesha, WI, USA).

All ultrasound procedures were performed with a Philips CX50 ultrasound machine (Philips Healthcare, Andover, MA, USA), using a 12-MHz linear array transducer with a 35-mm footprint, standard ultrasound gel, a 22-gauge, 89-mm diamond point stainless steel spinal needle (Covidien, Mansfield, MA, USA), and iodinated contrast agent (Omnipaque 300, GE Healthcare AS, Oslo, Norway) diluted with $0.9 \%$ saline to a concentration of $36 \mathrm{mg} / \mathrm{mL}$ $(10 \%)$. The injections were performed with the cadaver on the scanner table.

The TAP layer was identified along the midaxillary line by an ultrasound of each cadaver by an experienced sonographer. From superficial to deep, the external oblique muscle, internal oblique muscle, and transversus abdominis muscle, including the TAP fascial plane between the internal oblique and transversus abdominis muscles, were identified along the midaxillary line between the iliac crest and the subcostal margin, as previously described in the medical literature. ${ }^{4}$ A 22-gauge, 89-mm spinal needle was advanced under direct ultrasound visualization in a slightly oblique anterior to posterior direction toward the TAP. Once the needle tip was observed to enter this fascial plane, hydrolocation with $0.9 \%$ normal saline was performed to confirm the fascial plane and facilitate better sonographic visualization ${ }^{14}$ (Figure 1). Each cadaver was then placed in the CT scanner to verify the anatomical placement of the needle and to obtain baseline imaging of the relevant anatomy prior to contrast administration.

Following the initial CT scan, the needle was left in place and extension tubing with a syringe containing the dilute iodinated contrast agent was connected to the needle. Maintenance of needle position in the TAP layer was verified

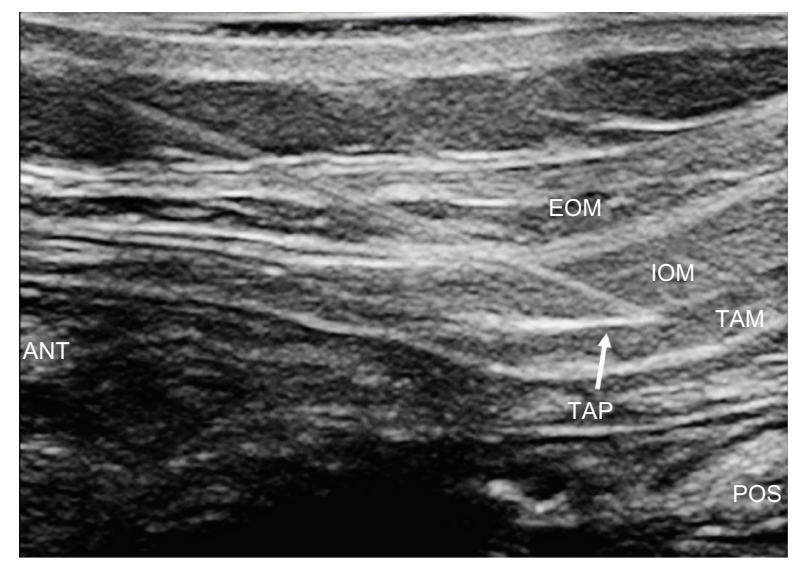

Figure I An ultrasound image of needle tip in the transversus abdominis plane (TAP) between the internal oblique muscle (IOM) and the transversus abdominis muscle (TAM) prior to injection. The external oblique muscle (EOM) is also well visualized. 
by ultrasound. A predetermined amount of contrast was then injected under direct ultrasound visualization into the TAP layer, and the cadaveric torso was rescanned using the same CT scan parameters. This procedure was repeated on both sides of each cadaver with different volumes of contrast: cadaver A (left, $5 \mathrm{~mL}$; right, $10 \mathrm{~mL}$ ); cadaver B (left, $15 \mathrm{~mL}$; right, $20 \mathrm{~mL}$ ). All CT scans were performed within 2 minutes of the contrast injection.

\section{Results}

All needle entry points were between the level of the lumbar (L)3 disk space and the middle of the L4 vertebral body. Contrast injected under ultrasound guidance was identified in the TAP in both cadavers on CT imaging (Figure 2 and 3). A small degree of intramuscular contrast was noted with each injection. Intraperitoneal contrast spread was also noted with the $15 \mathrm{~mL}$ injection (Figure 3).

Cranial-caudal spread of injected contrast correlated with increasing volumes of injectate (Table 1). The cranial-caudal distribution was roughly 1 vertebral level (end plate to end plate) for the $5 \mathrm{~mL}$ injection and 2 vertebral levels for the 10 , 15 , and $20 \mathrm{~mL}$ injections. Anterior-posterior and transverse spread of injected contrast did not correlate with increasing volumes of contrast (Table 1).

\section{Discussion}

As described in prior studies, there exists a difference in spread when a TAP block is performed under ultrasound guidance, versus a nonguided approach to the triangle of Petit. ${ }^{13}$ We chose to perform the injection using ultrasound guidance, with the goal of accurately measuring injectate

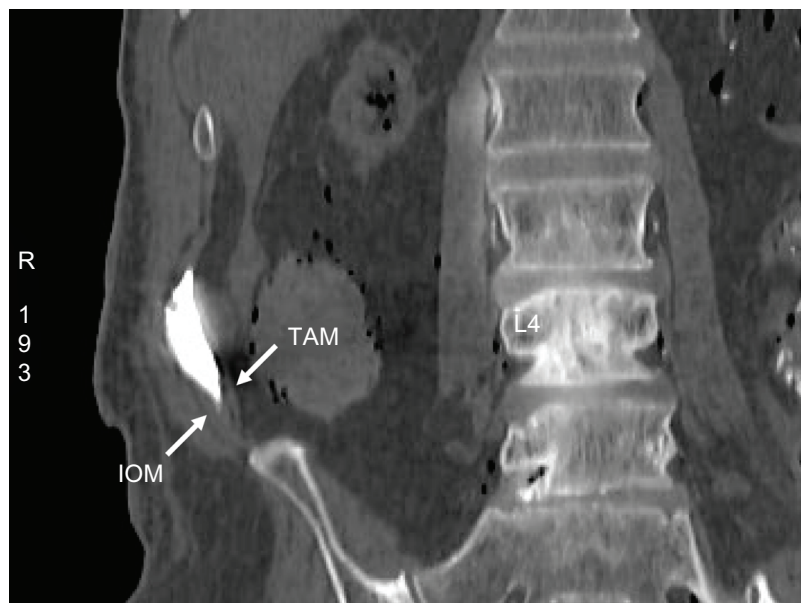

Figure $2 \mathrm{~A}$ coronal CT slice at the lumbar region demonstrating the spread of $5 \mathrm{cc}$ contrast material injected under ultrasound guidance into the transversus abdominis plane between the internal oblique muscle (IOM) and the transversus abdominis muscle (TAM).

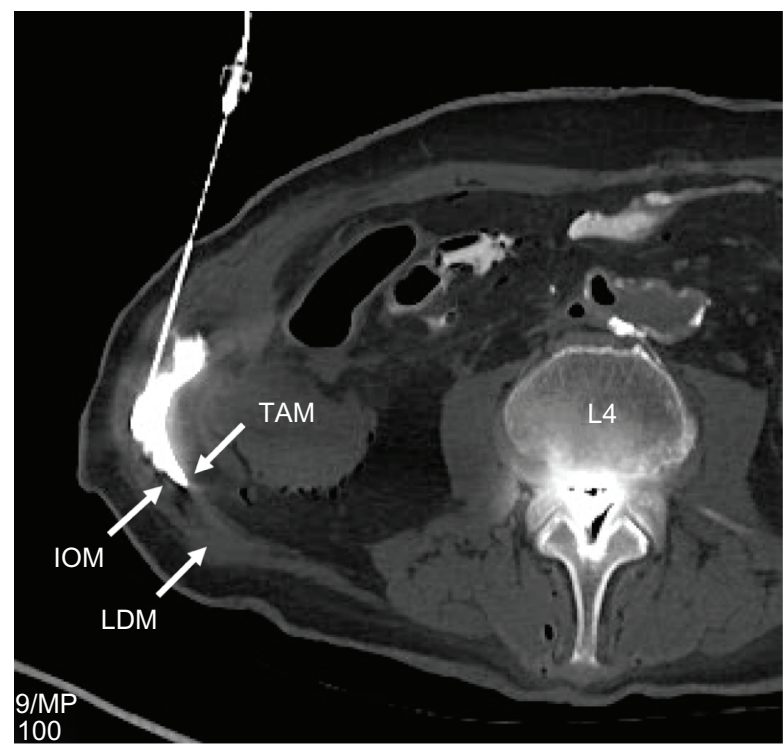

Figure 3 An axial CT slice at the level of the L4 vertebral body demonstrating the spread of $5 \mathrm{cc}$ contrast material injected under ultrasound guidance into the transversus abdominis plane between the internal oblique muscle (IOM) and the transversus abdominis muscle (TAM). The latissimus dorsi (LDM) is seen posteriorly.

spread delivered reliably into the transversus abdominis fascial plane. It is not uncommon to see a small amount of intramuscular spread of contrast within the TAP layer that is likely related to incomplete separation of the fascial planes under pressurization. However, we were surprised to discover contrast in the peritoneal space despite direct visualization of the needle placement with ultrasound. Logically, the ability to see the injection under live imaging guidance should be safer and more successful; however, the possibility of accessing an unintended target exists, regardless of experience or technique. ${ }^{15,16}$ The entrance of the needle and thus injectate could pose risk to patients including bowel penetration, injury, or infection. Furthermore, when performing injections on cadavers there is no movement such as breathing or bowel peristalsis which may make injections easier but can also make identifying structures more difficult. This is true for the TAP block, where the peritoneum can be identified

Table I lodinated contrast injectate spread of ultrasound-guided injections into the right and left transversus abdominis plane layers of two cadaveric torsos, as measured by computed tomography

\begin{tabular}{llll}
\hline $\begin{array}{l}\text { Injectate } \\
\text { volume, } \mathbf{~ m L}\end{array}$ & $\begin{array}{l}\text { Cranial-caudal } \\
\text { spread, } \mathbf{c m}\end{array}$ & $\begin{array}{l}\text { AP spread, } \\
\mathbf{c m}\end{array}$ & $\begin{array}{l}\text { Transverse } \\
\text { spread, cm }\end{array}$ \\
\hline 5 & 4.4 & 4.5 & 2 \\
10 & 6.1 & 1.6 & 3.4 \\
15 & 7.3 & 6.7 & 2 \\
20 & 8.4 & 1.7 & 5.8 \\
\hline
\end{tabular}

Abbreviation: AP, anterior-posterior. 
by movement and the muscle layer superficial to it can be identified as the transversus abdominis muscle followed by the internal and external oblique muscles.

Importantly, in this study, increased volumes of injectate correlated with increased cranial-caudal spread, and demonstrated good spread around the midaxillary line where the iliohypogastric, subcostal, and intercostal nerves have been shown to course in the TAP. ${ }^{1}$

\section{Conclusion}

This study helps further the understanding of injectate spread following ultrasound-guided TAP injections. Specifically, it suggests that $15 \mathrm{~mL}$ provides additional cranial-caudal spread and may be an optimal volume of anesthesia. The optimal volume required to achieve an adequate sensory block will vary, depending on the site of planned incision or the severity of abdominal pain. Furthermore, the degree of injectate spread may be different for live patients compared with that in fresh cadavers, and in addition, may also vary according to variability of patient height, weight, and previous abdominal surgeries. Therefore, further in vivo studies are warranted to investigate the minimum volume of ultrasound-guided injectate required to cause a sensory blockade of the anterior abdominal wall.

\section{Acknowledgments}

The authors would like to acknowledge the NIH construction grant, RR018898, (NIH C06 RR018898) which supported the radiology facility utilized in this study.

\section{Disclosure}

The authors report no conflicts of interest in this work.

\section{References}

1. Kanakarajan S, High K, Nagaraja R. Chronic abdominal wall pain and ultrasound-guided abdominal cutaneous nerve infiltration: a case series. Pain Med. 2011;12(3):382-386.
2. Bonnet F, Berger J, Aveline C. Transversus abdominis plane block: what is its role in postoperative analgesia? Br J Anaesth. 2009;103(4): 468-470.

3. Carney J, McDonnell JG, Ochana A, Bhinder R, Laffey JG. The transversus abdominis plane block provides effective postoperative analgesia in patients undergoing total abdominal hysterectomy. Anesth Analg. 2008;107(6):2056-2060.

4. El-Dawlatly AA, Turkistani A, Kettner SC, et al. Ultrasound-guided transversus abdominis plane block: description of a new technique and comparison with conventional systemic analgesia during laparoscopic cholecystectomy. Br J Anaesth. 2009;102(6):763-767.

5. McDonnell JG, Laffey JG. Transversus abdominis plane block. Anesth Analg. 2007;105(3):883.

6. Reid SA. The transversus abdominis plane block. Anesth Analg. 2007;105(1):282.

7. McDonnell JG, O’Donnell BD, Farrell T, et al. Transversus abdominis plane block: a cadaveric and radiological evaluation. Reg Anesth Pain Med. 2007;32(5):399-404.

8. Jankovic ZB, du Feu FM, McConnell P. An anatomical study of the transversus abdominis plane block: location of the lumbar triangle of Petit and adjacent nerves. Anesth Analg. 2009;109(3):981-985.

9. McDermott CD, Park J, Terry CL, Woodman PJ, Hale DS. Surgical outcomes of abdominal versus laparoscopic sacral colpopexy related to body mass index. J Obstet Gynaecol Can. 2012;34(1):47-56.

10. Hebbard P, Fujiwara Y, Shibata Y, Royse C. Ultrasound-guided transversus abdominis plane (TAP) block. Anaesth Intensive Care. 2007;35(4):616-617.

11. Hebbard PD, Barrington MJ, Vasey C. Ultrasound-guided continuous oblique subcostal transversus abdominis plane blockade: description of anatomy and clinical technique. Reg Anesth Pain Med. 2010;35(5): 436-441.

12. Børglum J, Jensen K, Christensen AF, et al. Distribution patterns, dermatomal anesthesia, and ropivacaine serum concentrations after bilateral dual transversus abdominis plane block. Reg Anesth Pain Med. 2012;37(3):294-301.

13. Abdallah FW, Chan VW, Brull R. Transversus abdominis plane block: a systematic review. Reg Anesth Pain Med. 2012;37(2):193-209.

14. Chin KJ, Perlas A, Chan VW, Brull R. Needle visualization in ultrasound-guided regional anesthesia: challenges and solutions. Reg Anesth Pain Med. 2008;33(6):532-544.

15. Horlocker TT, Wedel DJ. Ultrasound-guided regional anesthesia: in search of the holy grail. Anesth Analg. 2007;104(5):1009-1011.

16. Lancaster P, Chadwick M. Liver trauma secondary to ultrasoundguided transversus abdominis plane block. Br J Anaesth. 2010;104(4): 509-510.
Journal of Pain Research

\section{Publish your work in this journal}

The Journal of Pain Research is an international, peer-reviewed, open access, online journal that welcomes laboratory and clinical findings in the fields of pain research and the prevention and management of pain. Original research, reviews, symposium reports, hypothesis formation and commentaries are all considered for publication.

\section{Dovepress}

The manuscript management system is completely online and includes a very quick and fair peer-review system, which is all easy to use. Visit http://www.dovepress.com/testimonials.php to read real quotes from published authors. 